Rhode Island College

Digital Commons @ RIC

$12-1-2019$

\title{
Evaluating IV Acetaminophen for Pain Management After Cardiac Surgery: A Retrospective Chart Review
}

Jason Wilhelm

Follow this and additional works at: https://digitalcommons.ric.edu/etd

Part of the Nursing Commons

\section{Recommended Citation}

Wilhelm, Jason, "Evaluating IV Acetaminophen for Pain Management After Cardiac Surgery: A Retrospective Chart Review" (2019). Master's Theses, Dissertations, Graduate Research and Major Papers Overview. 320.

https://digitalcommons.ric.edu/etd/320

This Major Paper is brought to you for free and open access by the Master's Theses, Dissertations, Graduate Research and Major Papers at Digital Commons @ RIC. It has been accepted for inclusion in Master's Theses, Dissertations, Graduate Research and Major Papers Overview by an authorized administrator of Digital Commons @ RIC. For more information, please contact digitalcommons@ric.edu. 



\section{EVALUATING IV ACETAMINOPHEN FOR PAIN MANAGEMENT AFTER}

CARDIAC SURGERY: A RETROSPECTIVE CHART REVIEW

A Major Paper Presented

by

Jason Wilhelm

Approved:

Committee Chairperson

Committee Members

(Date)

(Date)

(Date)

Director of Master's Program

(Date)

Dean, School of Nursing 
Evaluating Ofirmev for Pain Management After Cardiac Surgery:

A Retrospective Chart Review

by

Jason Wilhelm

A Major Paper Submitted in Partial Fulfillment

of the Requirements for the Degree of

Master of Science in Nursing

in

The School of Nursing

Rhode Island College

2019 


\begin{abstract}
Pain after surgery is one of greatest complaints patients have in the surgical process. It is important to decrease pain after surgery to promote a quicker recovery and minimize complications. Opioids alone have been used to manage post-operative pain in cardiac surgery; However, recently multimodal approaches to pain management are now being explored. This approach involves using multiple medications with varying mechanisms of action for pain relief in addition to decreased adverse effects. Ofirmev (IV acetaminophen) is a relatively new medication for use in cardiac surgery that has few contraindications and side effects. The purpose of this study was to investigate if utilizing the current pain management approach in addition to Ofirmev impacted pain scores in post-operative cardiothoracic patients in a non-experimental retrospective chart review. A two group comparative chart review was conducted for a total of 30 charts to meet inclusion criteria. Group $1(n=15)$ received opioid only for pain management were compared to Group $2(\mathrm{n}=15)$ who received Ofirmev and opioids for pain management after cardiac surgery. Results showed that pain scores at hour 6 and 24 showed significance in favor of Group 2, the Ofirmev group. Results also showed Group 2, the Ofirmev group, consumed less morphine on average than Group 1, opioid only. Unexpectedly, length of stay was on average longer for the Ofirmev group than the nonofirmev group. The research supports the need to utilize multimodal pain management and alternative techniques to manage pain. This study shows there is a need for further research for pain management with a multimodal approach in cardiac surgery.
\end{abstract}




\section{Table of Contents}

Background/Statement of the Problem .................................................................. 1

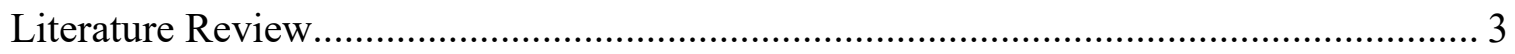

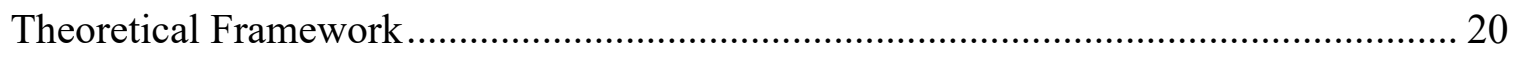

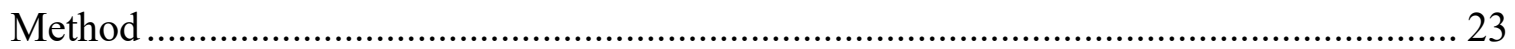

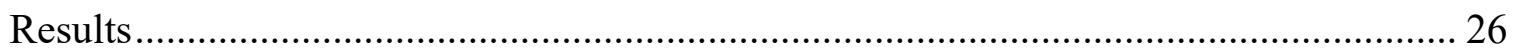

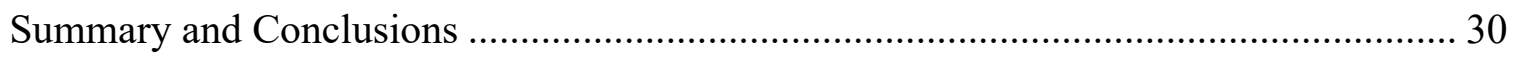

Recommendations and Implications for Advanced Nursing Practice .......................... 35

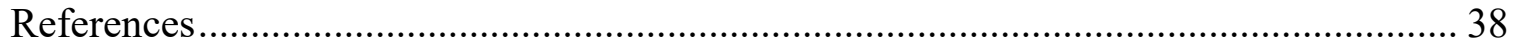

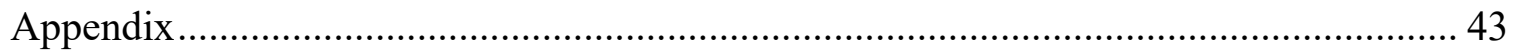




\section{Background/Statement of the Problem}

Pain is a subjective descriptor that many patients report experiencing, especially after surgery. Pain has many different pathways that contribute to the experience after surgery. Coronary bypass and valve replacement surgery are invasive surgeries after which many patients report feelings of pain. A primary cause of pain is related to the sternotomy, which is an incision made from the xiphoid process to the manubrium. Pain also develops due to sawing of the sternum as well as the retractors that are used to fully open the chest for surgery. After the procedure is performed, and prior to closing the wound, there are implantation of chest tubes, which further increases the painful experience. Due to the high level of pain associated with open heart surgery, this often leads to patients consuming an exorbitant amount of opioids on the path to recovery. While opioids may be effective in blocking one pathway for pain, they may introduce unwanted side effects. Side effects from opioids include: nausea, vomiting, drowsiness, respiratory depression, decreased blood pressure, paralytic ileus, and constipation. These unpleasant side effects can lead to delays in recovery or even detrimental complications.

Additionally, many patients are fearful of opioids due to the opioid epidemic that is currently the subject of significant media attention across the United States. According to the Center for Disease Control and Prevention ([CDC], 2018), opioids have killed more than 63,632 people in 2016 , with $66 \%$ of those deaths involving prescription opioids or an illicit drug. There are alternatives to using opioids for post-surgery that have less side effects, but these medications also have contraindications. For example, Ketorolac is a non-steroidal anti-inflammatory medication that can be used postoperatively, but it inhibits platelet function and may result in increased bleeding 
(Drugs.com, 2018). Recently Ofirmev (intravenous (IV) acetaminophen) has been introduced for use. Ofirmev has fewer side effects than opioids and less contraindications when compared to other non-opioid drugs. The Federal Drug Administration (FDA) approved the use of Ofirmev in November of 2010 (Drugs.com, 2018). Ofirmev reduces pain in a different pathway than opioids, however, Ofirmev's effectiveness in pain reduction still needs to be evaluated. Using a multimodal approach to pain control, including the administration of Ofirmev, may decrease the need for opioids in postoperative cardiothoracic patients. The purpose of this study was to investigate if utilizing the current pain management approach in addition to Ofirmev impacted pain scores in post-operative cardiothoracic patients in a non-experimental retrospective chart review.

Next is the review of the literature. 


\section{Literature Review}

An extensive literature review was conducted utilizing the databases of CINAHL, Ovid, Pub Med, and Medline. The search was limited to articles published in the English language from 2000 to 2018 to use updated references. Key words used for the literature review included: pain, cardiac surgery, cardiac, Ofirmev, Paracetamol, pain, multimodal pain, opioid addiction, cardiothoracic surgery, IV acetaminophen, and post-operative pain.

\section{Pain}

The International Association for the Study of Pain (IASP) defined pain as an "unpleasant sensory and emotional experience associated with actual or potential tissue damage, or described in terms of such damage"' (American Pain Society, 2018, p. 4). Pain works on a pathway called nociception. Nociception refers to the process by which information about tissue damage is conveyed to the central nervous system, but how this information is perceived as painful is unclear (American Pain Society, 2018). According to the American Pain Society (APS) the pain process works in multiple steps (2018). Nociceptors are sensory receptors that are sensitive to tissue trauma or a stimulus that would damage tissue if prolonged. These receptors are spread throughout the peripheral nervous system.

There are two steps in the process of nociception: transduction and transmission. Step one is transduction which occurs when injury to tissue causes cells to break down and release various tissue byproducts and mediators of inflammation, such as: prostaglandins, substance P, bradykinin, histamine, serotonin, cytokines (APS, 2018). 
These byproducts and mediators can activate the nociceptors that then generate a nerve impulse. Continuous activation of these nerve pathways may cause nociceptive pain. Transduction then leads to the second step of transmission, where nerve impulses generated in the periphery travel to the spinal cord and brain. Most impulses travel via nerve processes (axons) of primary afferent neurons to the dorsal horn (DH) of the spinal cord (APS, 2018). The nerve impulses are triggered through the release of excitatory amino acids (e.g. glutamate, aspartate) and neuropeptides (e.g. Substance P) at synapses between cells. It is important for health care providers, such as advanced practice registered nurses (APRNs) to understand this process, so they may interpret how medications can suppress pain during various steps of nociception. For example, acetaminophen is believed to inhibit cyclooxygenase enzymes and prostaglandin synthesis in the central nervous system. Although the complete mechanism of action is not fully understood, blocking cyclooxygenase enzymes and prostaglandins facilitate in blocking these two pain pathways (Douzjian \& Kulik, 2017). An APRN needs to understand how pain pathways work to choose medications which act on different mechanisms to maximize the benefit of blocking pain at multiple pathways.

Pain can be temporary, acute, or chronic. Acute pain usually appears suddenly because of disease, injury, or inflammation. Pain is a subjective, unpleasant sensory and emotional experience that varies from person to person. Acute pain is important as it serves as a biological function to warn of the potential for, or extent of, injury. Unfortunately, even brief intervals of pain can induce suffering, neuronal remodeling, and chronic pain associated behaviors (e.g. bracing, abnormal postures, excessive reclining). Having this knowledge has led to increased attention and focus on aggressive 
prevention and treatment of acute pain, which potentially will reduce complications, including progression to chronic pain states (APS, 2018). Chronic pain is recognized as pain that extends beyond the period of healing, with levels of identified pathology that often are low and insufficient to explain the presence and/or extent of the pain (APA, 2018). Persistent pain disrupts sleep and normal living, ceases to serve as a protective function, and ultimately degrades health and functional capability (APS, 2018). Unlike acute pain, chronic pain serves no adaptive purpose.

Pain is an unpleasant sensory and emotional experience that is associated with actual or potential tissue damage (Gebhart, 2000). There are two other types of pain: nociceptive and neuropathic. Neuropathic pain is now defined by the IASP as pain caused by a lesion or disease of the somatosensory nervous system. Neuropathic pain is caused by aberrant signal processing in the peripheral or central nervous system that reflects nervous system injury or impairment. Common causes of neuropathic pain include trauma, inflammation, metabolic diseases (eg. diabetes), infections, tumors, toxins, and primary neurological diseases (APS, 2018).

Nociceptive pain comes from the peripheral receptors as a result of injury and consists of somatic and visceral pain. The somatosensory nervous is known as conscious perception of touch, pressure, pain, temperature, position, movement, and vibration, which arise from the muscles, joints, skin, and fascia (Murnion, 2018). Somatic pain refers to injury to soft tissue, connective tissue, and bone. Usually this pain is described as sharp, localized discomfort (Salani, Crenshaw, Owusu, \& Gonzalez, 2018). Visceral pain refers to a deeper structure, such as an organ, that is usually described as dull, poorly localized, and sensitive to stretch (Salani et al., 2018). 


\section{Cardiothoracic Surgery and Pain}

Background. Every surgery has different types of pain, which can be traced to a certain aspect of the surgery itself. The pain is primarily due to surgical incisions and cauterization in the process necessary to perform every procedure. Unlike a common laparoscopic appendectomy which may require 3 small incisions to complete, cardiothoracic surgery involves a more invasive technique to the patient (Zubryzycki et al., 2018). This procedure is considered a traumatic surgery when using the standard median sternotomy approach, which deploys a saw to cut from xiphoid process to the suprasternal notch. This traumatic surgery causes superficial and deep somatic pain. Once the sternum is resected and chest retractors are placed, this surgery often requires cardiopulmonary bypass circulation. Cardiopulmonary bypass circulation is a process that allows the blood to be oxygenated and circulated to other organs in the circulatory system while the heart is in arrest. This process stimulates the systemic inflammatory response syndrome which contributes to inflammation at the surgical sites leading to pain (Fot et al., 2017). Chest tubes are placed strategically within the mediastinal and pleural cavities to drain excess fluid from accumulating around the heart and lungs. The phrenic nerve is located on the left and right sides of the heart, often close to the pleural chest tubes. Keeping this in mind, the surgeon needs to be careful to not irritate or compress either phrenic nerves. Nerve damage is regarded as one of the factors for acute and chronic pain in thoracic surgery, with chronic pain in $20 \%-80 \%$ of adult patients (Zubryzycki et al., 2018). This can often cause pain that radiates to the left shoulder as often there is a left pleural chest. This is characterized by the occurrence of allodynia and/or hyperalgesia, which suggests neuropathic pain in origin. Zubrzycki et al. also noted that psychological 
components of pain in addition to factors related to surgery have an impact on the sensation of pain (2018). These components would include high levels of anxiety, emotional lability, and pessimistic attitude.

\section{Pain Management}

Background. In 2012, the American Society of Anesthesiologists published guidelines for treating peri-operative pain based on the World Health Organization's analgesic ladder (Zubryzycki et al., 2018). These were made to increase the efficacy of acute, peri-operative pain management and improve the patients' quality of life through the process. The aim of effective post-operative analgesia is to provide the patient subjective comfort, facilitate the recovery process, and reduce the risk of complications. Zubryzycki et al. states that effective treatment should be multidimensional and based on three main therapeutic principles: administration of analgesics, multimodal analgesia, and regional anesthesia techniques (2018). This involves utilizing pharmacotherapy that works on pain in multiple pathways.

Opioids work through receptors in the central nervous system, including the $m u$, kappa, and delta opioid receptors. The principal receptors associated with pain physiology and inhibition are the $m u$ and kappa. Opioids bind to opioid receptors providing pain relief. Unfortunately, this relief comes with many negative side effects, including: constipation, decreased GI motility, nausea, hypotension, urinary retention, euphoria, pruritus, miosis, dependence, respiratory depression, and sedation (Salani et al., 2018). A common complication of peri-operative opioid use is a paralytic ileus. A paralytic ileus is an intestinal pseudo-obstruction that can cause signs and symptoms of 
intestinal obstruction, but does not involve a physical blockage. In paralytic ileus, muscle or nerve problems disrupt the normal coordinated muscle contractions of the intestines, slowing or stopping the movement of food and fluid through the digestive system, which is potentially caused by opioids (Mayo Clinic, 2018). A paralytic ileus may require surgery if symptoms are not reversed. These negative side effects lead to using alternative medications with lesser adverse effects in conjunction with opioids for pain management.

Evidence of Pain Management. Pharmacology is a common means to manage pain although it does not come without complication. In a study that investigated opioidinduced respiratory depression, which is a known cause of preventable death in hospitals, investigators analyzed inpatient hospitalization data of 14,504,809 medical inpatients and 6,771,882 surgical inpatient discharges (Izrailtyan, Qiu, Overdyk, Erslon, \& Gan, 2018). The goal of the study was to determine what specific factors increase the risk of inhospital cardiopulmonary and respiratory arrest (CPRA) in medical and surgical patients on opioid and sedative therapy (Izrailtyan et al., 2018). These patients were divided into four categories: on opioids, on sedatives, on opioids and sedatives, and on neither. Results showed that the vast majority of patients in the study ( $57 \%$ of all medical patients and $90 \%$ of all surgical patients) were prescribed opioids, sedatives, or both. The surgical patients with opioids had 13,282 occurrences of CPRA versus 3,474,484 without CPRA ( $\mathrm{p}$ value $<0.0001$ ). Medical patients with opioids had 13,775 patients with CPRA and $3,170,232$ patients without CPRA ( $\mathrm{p}$ value $<0.0001$ ). The study concluded that opioids and sedatives are correlated with cardiopulmonary resuscitation in both medical and surgical patients (Izrailtyan et al., 2018). Limitations of the study note that utilizing administrative databases capture only billing claims and may exclude clinical data. 
One alternative to pharmacotherapy for pain management is regional anesthesia. Regional anesthesia techniques are nerve blockers, which are in specific areas based upon the location of expected pain. Thoracic epidural analgesia is given continuously for cardiac and thoracic surgery to ensure effective control of intra and post-operative pain (Zubryzycki et al., 2018). However, this technique raises concerns in regard to the invasiveness and high cost.

Transcutaneous electrical nerve stimulation (TENS) is also used along with, or instead of post-operative analgesia to reduce demands for analgesic drugs, lower incidence of complications in the post-operative period, and possibly earlier ambulation. TENS is an alternative to pharmaceutical pain therapy but lacks research within the cardiothoracic post-operative patient population (Zubryzycki et al., 2018). Other nonpharmacological methods to alleviate post-operative pain may include relaxation, massage, heat therapy, acupuncture, hypnosis, reiki and cognitive-behavioral therapy. While empirical evidence to support the use of these alternative methods is limited, some initial research shows signs of effectiveness in the cardiothoracic post-operative patients (Zubryzycki et al., 2018). For example, Babaee, Shafiei, Sadeghi, Nik and Valiani (2012) found post-operative massage improved patients' overall mood after surgery. Mood was characterized by assessing: anxiety, depression, anger, ability, fatigue, and confusion in the questionnaire. These investigators performed a randomized single-blind clinical trial with patients undergoing cardiothoracic surgery. There were 72 patients included in the study who were divided into a control group and an intervention group. The intervention group received Swedish massage for 20 minutes during four sessions over four consecutive days, between post-operative day three to post-operative day six (Babaee et 
al., 2012). A mood questionnaire was used prior to surgery and again after the last day of the intervention. The questionnaire covered moods such as anxiety, depression, anger, ability, fatigue, and confusion. The mean for all the categories compiled before intervention was 123.1 and decreased to a mean of 23.1 after the massage intervention. The findings of the study showed significant mood rating changes in all categories $(p<0.001)$ (Babaee et al., 2012). This study shows that different forms and techniques of pain management can be effective and beneficial. The next section will discuss various types of pain management in the post-operative population.

\section{Post-Operative Pain Management}

Background. More than $80 \%$ of patients undergoing surgery experience postoperative pain, and $75 \%$ of those patients rate their pain as moderate, severe, or extreme (Chou et al., 2016). The American Pain Society has a panel of 23 members with expertise in anesthesia and/or pain medicine, surgery, obstetrics and gynecology that create a composite of guidelines for post-operative pain management. Currently, the panel recommends use of a variety of analgesic medications and techniques, combined with non-pharmacological interventions for the treatment of post-operative pain (Chou et al., 2016). Some non-pharmacological interventions include Reiki, warm compress, ice, therapeutic touch, and relaxation techniques (Chou et al., 2016). Acetaminophen and NSAIDs in conjunction with opioids are associated with less post-operative pain and decreased opioid consumption due to their different mechanisms of action (Chou et al. 2016). Ineffective pain management can increase the risk of postsurgical complications, which can lengthen hospitalization (Kwan, 2017). It is important to understand the 
process of recovery with regards to pain management to minimize suffering while having a better recovery after surgery.

\section{Evidence of Pain Management in Post-Operative Cardiac Surgery Patients.}

Nachinynde and Lam (2018) reviewed randomized controlled trials involving adults eighteen years and over having post cardiac surgery. Their review included the use of subcutaneous anesthetic infusions, paravertebral blocks/infiltrations, patient-controlled analgesia (PCA), Ketamine/opioid infusions and nurse administered subcutaneous opioids for pain management in the intensive care unit. Their search yielded 1875 articles, but inclusion and exclusion criteria narrowed this to ten articles. The articles had a total of 511 patients included for the final analysis. Local infiltrations and parasternal blocks were more effective in the first 4-6 hours and up to 24 hours post-operatively. Continuous subcutaneous anesthetic infusions were effective much longer and associated with reduced length of stay. The use of local anesthetic infusions with PCA demonstrated superior analgesia to PCA alone in reducing pain in minimally invasive cardiac surgery (e.g. a non-sternotomy approach). Ketamine was used successfully for pain management in four trials and demonstrated that it reduced opioid consumption in post-cardiac surgery, but in post-knee arthroscopy, Ketamine failed to improve on analgesia or decrease opioid consumption rendering it inconclusive (Nchinyde \& Lam, 2018). Limitations in this study included limited number of patients for analysis, lack of reported outcome measures by some studies like complications and length of stay. The study concluded that PCA with opioids in conjunction with subcutaneous anesthetic continuous infusions was more effective longer. There is a need for caution with Ketamine use in post-cardiac surgery until more dose-related studies are done (Nchinyde \& Lam, 2018). 
In another study by Kumar et al. (2018), investigators compared the use of a pectoral nerve block with ultrasound in patients undergoing cardiac surgery. The sample included a total of 40 participants, who had a sternotomy incision approach. Twenty participants received standard care in the control group and 20 received the pectoral nerve block. All the patients received scheduled paracetamol IV and tramadol 50mg IV twice a day. Group numbers were concealed in sealed opaque envelopes that were opened after patients joined the study. After surgery they recovered in the ICU. Once the patient was hemodynamically stable in the ICU and the local anesthetic was given, the procedure was performed with the ultrasound probe. An unnamed local anesthetic of 20 milliliters solution was administered between pectoralis minor and serratus anterior muscles. This procedure was performed on both sides of the chest. Pain was measured using the visual analog scale (VAS) with pain recorded at $0,3,6,12,18$, and 24 hours. The control group pain scores recorded at $0,3,6,12,18$, and 24 hours were $4.45,3.8,4.4,3.6,3.3$, and 3 , respectively. The interventional group pain scores at $0,3,6,12$, and 18 hours were 1.45 , $1,11.15,1.55,1.85$, and 2.85 , respectively. The interventional group pain scores were found to be significantly lower in comparison to the control group for the first 18 hours after extubation $(p<0.05)$ (Kumar et al., 2018). This study provides evidence that alternative pain management techniques in the cardiothoracic patient population, such as a pectoral block is a simple, safe, and effective technique (Kumar et al., 2018).

IV Acetaminophen use. Acetaminophen is an antipyretic and analgesic agent, and it is more commonly used to relieve mild to moderate pain caused by different etiologies. The IV form of acetaminophen was approved and made available in 2010 (e.g. Ofirmev). The new availability of IV acetaminophen has allowed for a multimodal 
approach to pain management (Waknine, 2010). For patients in need of critical care, Ofirmev has less complications while being injected, as well as post-administration, when compared to IV morphine sulfate. Some side effects of morphine include: respiratory depression, constriction of common bile duct and spams of the sphincter of Oddi, drowsiness, sedation, dry mouth constipation, nausea, diarrhea, anorexia, vomiting, and urinary retention (Drugs.com, 2018).

Arslan, Celep, Ciçek, Kalender, and Yilmaz (2013) investigated the use of IV paracetamol (acetaminophen/APAP) and its effects during the peri-operative period in patients undergoing a cholecystectomy. In this randomized control study, group I was given IV paracetamol prior to surgery, group II received IV paracetamol at the end of the operation, and group III received $100 \mathrm{ml}$ saline at the end of the surgery (Arslan et al., 2013). A satisfaction questionnaire was done 24 hours after surgery with options to rate from poor to excellent. Side effects of nausea, vomiting, itching, respiratory depression, allergic reaction, rash, stomach irritation, diarrhea, constipation, drowsiness, headache, dry mouth, sweating, and hypotension were recorded. Groups I and II had significantly lower need for supplemental analgesics compared to group III with a $(p<0.05)$. Group I also had significantly lower need for supplemental analgesics compared to group II $(p<0.05)$. The incidence of nausea and vomiting was greater in groups II and III $(p<0.05)$ (Arslan et al., 2012). The researchers concluded that early post-operative analgesia with 1 gram $(\mathrm{g})$ of paracetamol in patients undergoing cholecystectomy provided decreased opioid consumption with lower side effects. Findings demonstrated significant evidence of lower total opioid consumption during the 24 hour post-operative period for group I $(p<0.001)$ and group II $(p<0.001)$ when compared to group III. Analgesic consumption 
(tramadol $\mathrm{mg}$ ) total was as follows (Mean \pm Standard Deviation) group I: 60.0 \pm 76.5 , group II 103.0 \pm 85.8 , and group III 182.0 \pm 93.6 (Arslan et al., 2013). The authors also point out that there is strong evidence in the literature demonstrating that paracetamol can be used safely during post-operative pain management (Arslan et al., 2013).

Intravenous paracetamol has also been investigated for pain relief in gynecological surgeries. Mohamad, McDonnell, Bloor, Nathan \& Paech (2014) hypothesized that pain caused from the release of prostaglandins could be relieved by parecoxib and paracetamol. In a randomized, blinded, placebo-controlled multi-group study, investigators compared the use of IV paracetamol (APAP) to Parecoxib (COX 2 inhibitor, e.g. Celebrex) in 240 women undergoing dilatation and curettage. Participants were divided into four groups: group A (2g IV Paracetamol), group P (40 milligrams IV Parecoxib), group AP (both Paracetamol and Parecoxib), and group C (IV saline, placebo). Although the researchers noted that the results of the study were inconclusive, they speculated that the multi-modal medication approach may benefit a major surgery (abdominal or orthopedic) that causes more deep tissue damage or injury (Mohamad et al., 2014). This study was limited to minor gynecological surgery (hysteroscopy and uterine curettage) which may have less inflammatory and neuroendocrine response in comparison to a more traumatic surgery (Mohamad et al., 2014).

In a retrospective chart review, researchers evaluated whether the use of Ofirmev reduced the intake of opioids in total hysterectomy patients (Herring et al., 2014). The population consisted of 100 patients from one tertiary care community hospital. Patients were divided into two groups: a standard care group of 50 patients who received opioids only and a multimodal group of 50 patients who received IV acetaminophen every six 
hours in addition to opioids. The results of the study revealed a $31 \%$ decrease of opioid use in the multimodal group as opposed to the opioid only group. An average of 99 milligrams (mg) of morphine equivalents was used in the opioids-only group versus an average of $73 \mathrm{mg}$ of morphine equivalents in the multimodal group $(p=0.001)$ (Herring et al., 2014). Limitations in this study included no power calculation to determine appropriate sample size and the investigation of only one type of surgical procedure.

Overall, researchers concluded that post-operative, and peri-operative, use of intravenous acetaminophen as an adjunct analgesic in patients undergoing total abdominal hysterectomy procedure was effective in providing opioid sparing effects (Herring et al., 2014).

A randomized, double-blinded, placebo-controlled study conducted by Strode et al. (2015) studied the effects of Ofirmev administration versus a placebo for pain management in patients undergoing laparoscopic gastrectomy. Researchers aimed to determine if opioid consumption could be reduced with the additional use of Ofirmev in surgical patients following laparoscopic gastrectomy sleeve. The control group received a placebo and fentanyl via patient controlled analgesia, while the experimental group received Ofirmev 1 gram IV with fentanyl via patient controlled analgesia. This study took place in a United States (U.S.) military training hospital and included 34 patients. A total of 18 patients were assigned to the Ofirmev group and 15 assigned to the placebo group. In the 24 hours following surgery, Ofirmev was scheduled every six hours, as was the placebo (normal saline) in the control group. The visual analog scale (VAS) was used to determine pain levels every four hours for 24 hours following surgery. Using a line graph, the authors identify that pain scores at 12,16 , and 20 hour point times in the 
Ofirmev group were significantly lower than that of the control group $(p=.02, .03$ and .01 , respectively), but the amount of opioid use did not decrease in the population in comparison to the control group (Strode et al., 2015). This study was limited by a small sample size of only 35 patients. Additionally, one factor that was not controlled for was PCA use. This may have impacted the study by altering pain levels, thus skewing the pain scale results.

Apfel, Jahr, Kelly, Ang and Oderda (2015) compared IV acetaminophen in conjunction with the typical opioid regimen versus opioids only for pain relief in postoperative orthopedic surgical patients. The purpose of the study was to investigate the relationship between non-opioid medications used for pain relief in comparison to adverse events, hospital length of stay (LOS), and overall hospital costs. This design was a retrospective cohort study of case-matched patients who underwent elective total hip arthroplasty or total knee arthroplasty, from 550 hospitals. Case-matching included two populations: one for each case who received IV acetaminophen on the day of surgery paired to another that did not receive IV acetaminophen within the same hospital. There was a total of 22,146 cases and the researchers controlled for age, race, sex, marital status, insurance status, and pre-operative comorbidities. Adverse events were significantly lower in the IV acetaminophen group ( $n=2688,24.3 \%)$ in comparison to the control group $(n=2913,26.3 \%)(p<0.001)$. The LOS was also decreased in the IV acetaminophen group in comparison to the control group (2.96 days versus 3.04 days, $p<$ 0.001). Costs between the two groups also varied with an average of $\$ 16,381$ in the IV acetaminophen group in comparison to the control group costs of $\$ 16,927(p<0.001)$. The researchers, however, were unclear in their reporting of the procedures for IV 
acetaminophen administration, only reporting that patients included were those who received a dose of IV acetaminophen on the day of surgery. The intervention group was not described with respect to the amount, frequency, or timing of administration (pre-, post-, intra-, or peri-operative periods). Limitations of this study are that the findings may not be applicable to other surgeries. In this retrospective cohort study using multimodal analgesia with IV acetaminophen, there was an association with improved clinical outcomes. Those patients who received IV acetaminophen were found to have fewer adverse events, shortened length of stay, and reduced total hospital resources compared with patients not receiving IV acetaminophen (Apfel et al., 2015).

Despite advancements and improvements in pain management over the years, a gap of post-operative management still exists. Overall, the post-operative patient population has shown the need to change the approach for pain management.

IV Acetaminophen use in Cardiothoracic Patients. Although Ofirmev has only been available since 2010, there have been multiple peri-operative studies. There is, however, minimal research in cardiothoracic surgical patients which leads to the one available study by Rafiq et al. (2014). Rafiq et al. (2014) conducted a prospective randomized controlled trial including 180 patients between March 2007 and August 2009 for cardiac surgery. The population came from a single center where patients were approached about the study on the day prior to cardiac surgery and randomized after consent with sequentially numbered envelopes to one of two groups. Exclusion criteria included peripheral neuropathy, neurological disease, psychiatric illness, history of GI bleeding, chronic pain, hepatic disease with elevated liver enzymes, allergy to study 
medications, alcohol abuse, abuse of narcotics, pregnancy, and insufficient language skills.

This study's objective was to find an alternative medication regimen for cardiac surgical patients in comparison with the typical opioid regimen, because of the adverse opioid-related effects. The alternatives included gabapentin, dexamethasone, ibuprofen, and acetaminophen scheduled in a protocol driven alternative to morphine alone (Rafiq et al., 2014). The intervention group (multimodal group) received ketorolac, paracetamol (acetaminophen), gabapentin, ibuprofen, and dexamethasone, while the control group receiving morphine and paracetamol only. Pain was rated using the numbers scale for the primary outcome focus of the study. The relationship of the multimodal protocol that was implemented contained multiple medications that directly target pain receptors in different pathways (Rafiq et al., 2014). Gabapentin acts mainly on inhibiting central neuronal sensation. Ketorolac and ibuprofen are NSAIDS that block the prostaglandin synthesis. Dexamethasone is a corticosteroid that has anti-inflammatory properties. Paracetamol a prostaglandin inhibitor (Rafiq et al., 2014).

The results of this study show that the average pain difference between the multimodal group and the morphine only was significant on days 1, 2, and 3. Pain was broken down into worst, least and average pain scores. On day one, the worst pain scores category for the multimodal group had a mean $(\mathrm{M}) \pm$ standard deviation (SD) of 4.6 \pm 2.4 , while the morphine group had a $\mathrm{M} \pm \mathrm{SD}$ of 5.5 \pm 2.4 (Rafiq et al., 2014). On day one, the least pain scores category for the multimodal group had a $\mathrm{M} \pm \mathrm{SD}$ of $1.6 \pm 1.0$, while the morphine group had a $\mathrm{M} \pm \mathrm{SD}$ of $2.1 \pm 1.2$ (Rafiq et al., 2014). On day two, the worst pain scores category for the multimodal group had a pain scores category for the multimodal 
group had a $\mathrm{M} \pm \mathrm{SD}$ of $1.7 \pm 2.4$, while the morphine group had a $\mathrm{M} \pm \mathrm{SD}$ of $2.5 \pm 1.7$. On day three, the worst pain scores category for the multimodal group had a $\mathrm{M} \pm \mathrm{SD}$ of 3.6 \pm 2.4 , while the morphine group had a $\mathrm{M} \pm \mathrm{SD}$ of $3.9 \pm 2.3$. On day three, the least pain scores category for the multimodal group had a $\mathrm{M} \pm \mathrm{SD}$ of $1.3 \pm 0.8$, while the morphine group had a $\mathrm{M} \pm \mathrm{SD}$ of $1.8 \pm 0.8$ (Rafiq et al., 2014) ( $p=0.001$ ). Though the difference between the two groups was not quite as large on day 3, findings were still significant ( $p=0.032$ ) (Rafiq et al., 2014). Although this study included multiple medications in the experimental group, which make it impossible to determine which medication had the greatest impact on the reduced pain outcomes, the researchers provide evidence that a standard morphine only regimen does not maximize pain management. Implementing a regimen with different medications that work on multiple pathways to decrease pain has the potential to improve outcomes, as well as satisfaction in cardiac surgical patients. It is important to further explore the combinations and available techniques to decrease pain after cardiac surgery for the best outcomes possible.

Next, the theoretical framework will be discussed. 


\section{Theoretical Framework}

\section{Theory of Unpleasant Symptoms}

The theory of unpleasant symptoms (TOUS) was developed by four nurse researchers who focused on identifying commonalities in nursing that integrated different symptoms experienced by various populations in different scenarios (Smith \& Lehr 2008). This theory is based on different symptoms, and their similarities, or shared negative experiences. The theory inspects the relationships of populations that are affected from outside factors and the symptoms that preside from the scenario. Gift, Milligan, Pugh and Lenz each worked on different symptoms. Gift studied dyspnea, while Milligan and Pugh studied fatigue, and Lenz collaborated on various empirical studies and theoretical articles. Working at the same university, they shared common exposure to the same philosophical and meta-theoretical perspectives regarding the development and substance of nursing science to address symptoms and their outcomes (Smith \& Lehr, 2008).

TOUS is based on the interactions of symptom(s), influencing factors, and performance outcomes. The influencing factors are categorized as physiologic, psychologic, and situational that interact within each other to influence the symptom(s). According to Smith and Lehr (2008), the symptom(s) experience, in turn, affects the individual's performance, which encompasses cognitive, physical, and social functioning. This cycle of a theory shows that an individual's performance (life equilibrium) can be affected by unpleasant symptoms that may also be affecting physiological, psychological, and situational factors. Unpleasant symptoms are utilized in this theory to explain the relationships of multiple factors for any given person, as depicted in Figure 1. 


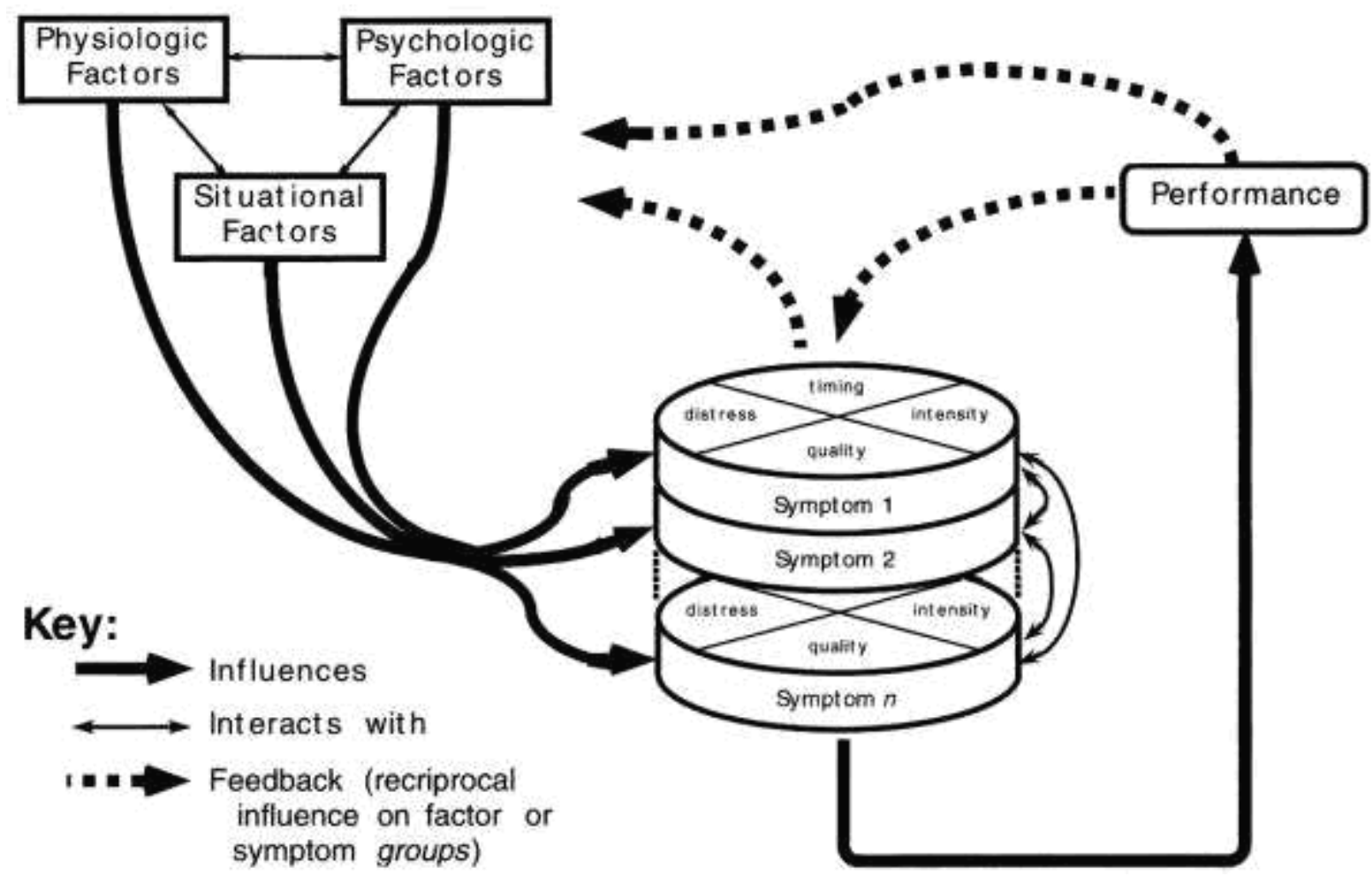

Figure 1. Middle Range Theory of Unpleasant Symptoms (Smith \& Liehr, 2008)

The non-specific, but applicable concept of TOUS, is applicable to numerous patient populations. Woods, Kozachik, and Hall (2010) utilized the framework in a study to measure sleep quality for women currently or previously in an abusive relationship. The hypothesis predicted that psychological factors of PTSD, physiological symptoms of self-reported physical health symptoms, and situational factors of childhood maltreatment and intimate partner violence, all led to poor sleep quality (Woods et al. 2010). The relationships of PTSD to global sleep quality and disruptive nighttime behaviors were positive and statistically significant, with $\mathrm{p}<.01$, indicating greater PTSD severity was associated with poorer sleep quality. Likewise, French, Crawford, Bova and Irwin (2017) framed their study using TOUS as well. These researchers hypothesized that addressing anxiety and depressive mood disorders would improve chronic cough severity and quality 
of life. This was a longitudinal observational study of adult patients seeking care of chronic cough $>8$ weeks duration. The subjects attended an initial cough clinic visit that standard of care was based on American College of Chest Physician guidelines. Followup medical care was delivered in person or by telephone. The successful treatment of the chronic cough showed significant results $(p<0.05)$ with reduction in psychological symptoms over 3 months (French, Crawford, Bova \& Irwin, 2017).

Pain as previously discussed is a symptom that can have many negative affects that fit in the TOUS ideals. Using TOUS as a theoretical framework to study the effects of Ofirmev in the cardiothoracic surgery patient may demonstrate a positive impact on pain management in this population. Introducing a multimodal regimen of IV acetaminophen and typical opioids for management of pain is anticipated to decrease the unpleasant symptoms related to surgery. TOUS is an appropriate framework for analyzing the unpleasant symptoms and showing a relationship between decreased pain and its positive effects with performance (Polit \& Beck, 2017).

Next the method section will be discussed. 


\section{Method}

\section{Purpose}

The purpose of this study was to investigate if utilizing the current pain management approach in addition to IV acetaminophen effected pain scores in postoperative cardiothoracic patients in a non-experimental retrospective chart review.

\section{Design}

The design for this project was a retrospective two group comparative chart review, which investigated pre and post implementation of Ofirmev administration. The aim was to determine if there was a difference in pain scores between patients receiving adjuvant Ofirmev versus those receiving opioids only.

\section{Sample}

The sample for this project was derived from medical records, including both males and females, 18 years of age and older, in which cardiac surgery with full sternotomy was complete. The sample was separated into two groups of cardiac surgical patients. The interventional group, Group A, consisted of 15 cardiothoracic surgical patients, post-implementation of the Ofirmev protocol for surgical pain management in September 2017. The standard group, Group B, was chosen from 15 cardiothoracic surgical patients from the year prior to Ofirmev being available in the facility. Exclusion criteria included anyone with a history of opioid use for chronic pain, history of substance or opioid abuse, and history of dementia. 


\section{Site}

The site for this study was a 666-bed community hospital in the northeast United States. The population was derived from the CVICU, an eight-bed intensive care unit.

\section{Procedure}

Administrative approval was obtained from the Chief Nursing Officer as well as the research coordinator, and the CVICU unit manager at Charlton Memorial Hospital $(\mathrm{CMH})$. Although the site for this project does not have an internal review board (IRB), the project was approved by Rhode Island College's internal review board.

Thirty participants were randomly chosen through chart review of patient records who received cardiothoracic surgery. The records reviewed from January 2017 through October 2018, until a target sample size of 30 was met. A new protocol was implemented for administration of Ofirmev during September 2017 which required that Ofirmev be given upon preparation for extubation of patient from the ventilator, after surgery in the CVICU. This protocol is now employed for the first 24 hours after extubation. The administration of IV Ofirmev is scheduled every six hours for 24 hours total then discontinued. Records were retrieved for patients who received coronary artery bypass grafting or prosthetic heart valve replacement. All collected data was kept in a locked drawer of a locked nurse manager's office and was destroyed using a confidentiality shredder after completion of chart review. All analyses were conducted in accordance with HIPAA, utilizing a password protected laptop that only the researcher has access to. 


\section{Measurements}

A researcher designed data collection tool (Appendix A) was used, which included age, morphine equivalents administered, pain scores at: 0, 6, 10, 16, and 24 hours from first administration of Ofirmev, and length of stay. The numeric rating scale (NRS) was used to represent numerical value, 0 for no pain ranging to 10 for the most pain. The pain scores were recorded after the first dose of Ofirmev, which is administered while on weaning mode from mechanical ventilation in preparation for extubation. Data was collected using documented pain levels at 0 hours from first administration of Ofirmev, 6 hours, 10 hours, 16 hours and 24 hours. An opioid administration total was recorded for this 24 hour period and converted to morphine equivalents to quantify data. The administration of morphine equivalents was compared between the two groups to evaluate for differences in consumption for pain management. Patient demographic characteristics recorded were age and sex.

\section{Analyses}

Data was analyzed using descriptive statistics, including mean, median, and range. The total amount of opioids administered was also calculated to compare the two groups. Pain scores, morphine equivalence and length of stay by group were compared using a student's $t$-test. 


\section{Results}

Of the total of 128 charts that were reviewed, thirty charts met inclusion criteria, with fifteen in each group. Records were excluded based on exclusion criteria or incomplete information. The demographics of age for each group are included in Figure 2.

Figure 2. Distribution of Cases

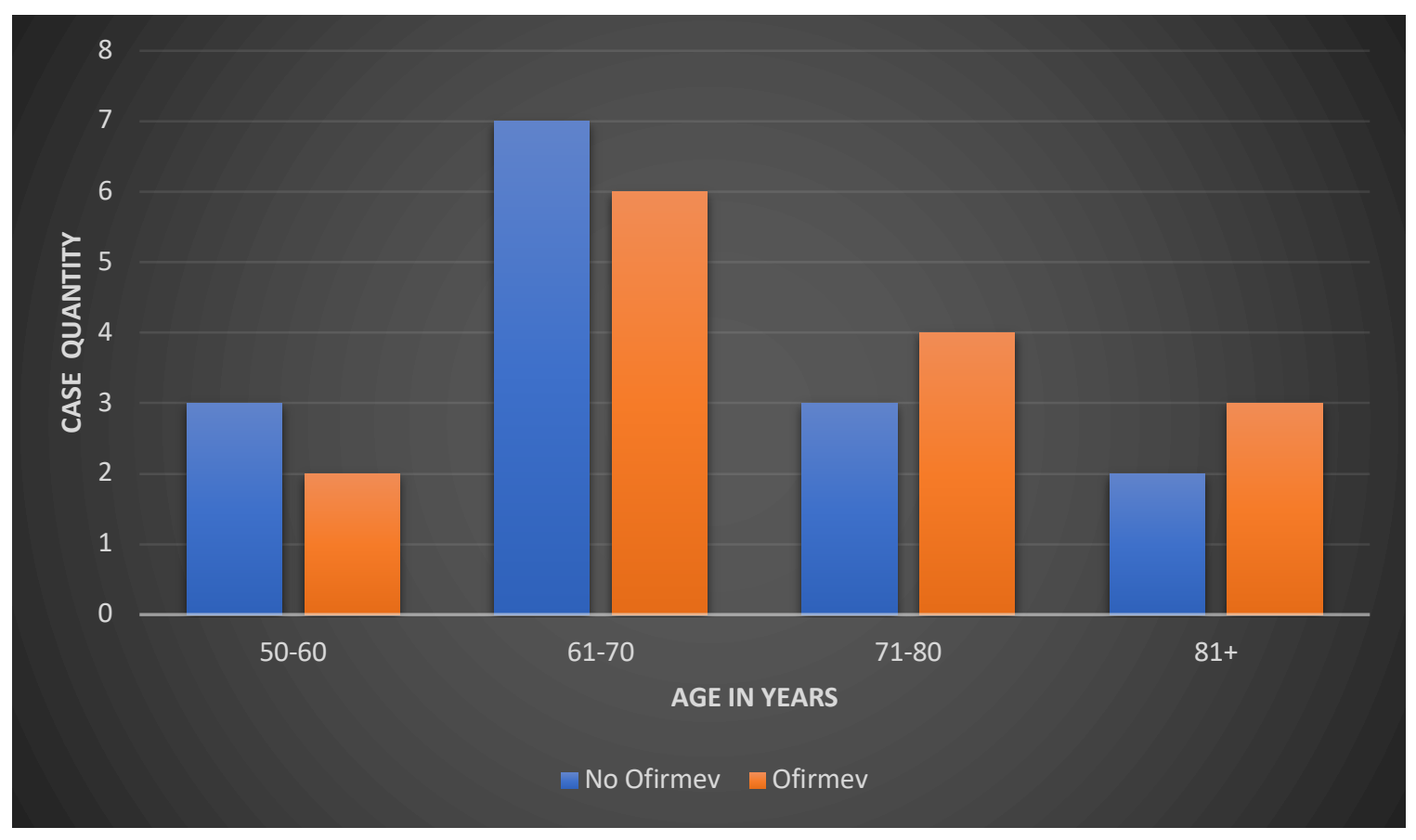

Of all of the cases in the project $(\mathrm{N}=30), 66.6 \%(n=20)$ were male and $33.3 \%$ $(n=10)$ were female. Approximately $83 \%$ of the cases $(n=25)$ were 61 years or older with about $16 \%(n=5)$ were less than 60 years of age. 
Figure 3. Mean Pain Scores Comparison

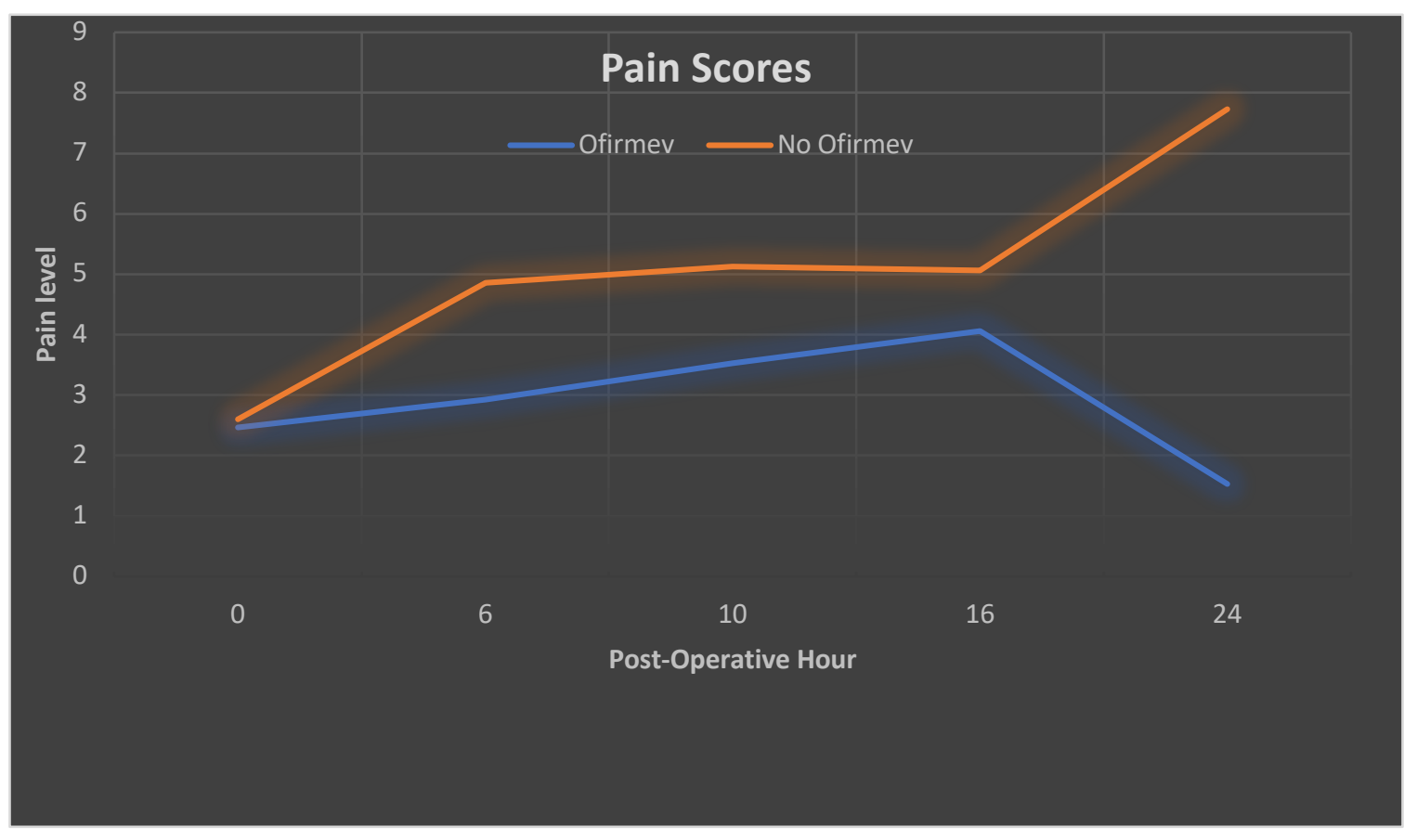

A comparison shows minimal difference in pain scores between the two groups up to hour 16 (Figure 3). At the zero hour time point, the pain scores for the non-ofirmev group $(M=2.60 ; S D=3.14, n=15)$ and Ofirmev group $(M=2.47, S D=3.36, n=15)$ were not found to be significantly different $(t(28)=0.11, p=0.46)$. However, at the 6 hours postoperative time point (Table 1), the pain scores in the non-ofirmev group ( $M=4.87$; $\mathrm{SD}=3.00, \mathrm{n}=15$ ) were found to be significantly higher than the pain scores of the Ofirmev group $(\mathrm{M}=2.93 ; \mathrm{SD}=3.10, \mathrm{n}=15)(\mathrm{t}(28)=1.74, p=.05)$. The 10 hour post-operative mark showed no significance in pain scores with Ofirmev $(\mathrm{M}=3.53 ; \mathrm{SD}=2.90, \mathrm{n}=15)$ compared to non-ofirmev $(\mathrm{M}=5.13 ; \mathrm{SD}=3.29, \mathrm{n}=15)(\mathrm{t}(28)=1.41, p=0.08)$, but approached significance. The 16 hour post-operative mark lacked any significance between the Ofirmev $(\mathrm{M}=4.07 ; \mathrm{SD}=1.91, \mathrm{n}=15)$ and non-ofirmev $(\mathrm{M}=5.07 ; \mathrm{SD}=3.13, \mathrm{n}=15)$ $(\mathrm{t}(28)=1.06, p=0.15)$. The only other significant pain score noted was at the twenty-four 
hour mark (Table 2). At 24 hours post-operatively, the pain scores in the non-ofirmev group $(M=4.13 ; S D=2.39, n=15)$ were found to be significantly higher than the pain scores in the Ofirmev group $(\mathrm{M}=1.53 ; \mathrm{SD}=1.51, \mathrm{n}=15)$ at 24 hours post-operatively $(t$ $(28)=3.57, p=.00)$.

Table 1. Pain Scores at 6 Hours Post-Operative

\begin{tabular}{|lrr|}
\hline Pain Scores 6hrs Post-Operative & & \\
\hline & NON-Ofirmev & Ofirmev group \\
\hline Mean & $\mathbf{4 . 8 7}$ & $\mathbf{2 . 9 3}$ \\
Observations & 15.00 & 15.00 \\
Df & 28.00 & \\
t Stat & 1.74 & \\
P(T<=t) one-tail & $\mathbf{0 . 0 5}$ & \\
\hline
\end{tabular}

Table 2. Pain Scores at 24 Hours Post-Operative

\begin{tabular}{|lrr|}
\hline Pain Scores 24hrs Post-Operative & & \\
\hline \multicolumn{1}{|c|}{ NON-Ofirmev } & Ofirmev group \\
\hline Mean & $\mathbf{4 . 1 3}$ & $\mathbf{1 . 5 3}$ \\
Observations & 15.00 & 15.00 \\
Df & 28.00 & \\
t Stat & 3.57 & \\
$\mathbf{P}(\mathbf{T}<=\mathbf{t})$ one-tail & $\mathbf{0 . 0 0}$ & \\
\hline
\end{tabular}

The mean scores for morphine equivalent consumption in the first twenty-four hours were $(58.77 \mathrm{mg})$ in the non-ofirmev group and $(44.07 \mathrm{mg})$ in the Ofirmev group (Table 3). Although there was no significance $(p=0.08)$, the difference between nonofirmev and Ofirmev approached significance. Interestingly, the length of stay (Table 4) in the non-ofirmev group $(\mathrm{M}=7.20 ; \mathrm{SD}=3.12, \mathrm{n}=15)$ was found to be significantly lower than the length of stay in the Ofirmev group $(M=11.40 ; S D=5.93, n=15),(t(28)=-2.43$, $p=.01)$. 
Table 3. Morphine Equivalence Comparison

Morphine Sulphate administration (in mg) 24hrs Post-Operatively

\begin{tabular}{|lrr|}
\hline & NON-Ofirmev & Ofirmev group \\
\hline Mean & $\mathbf{5 8 . 7 7}$ & $\mathbf{4 4 . 0 7}$ \\
Observations & 15.00 & 15.00 \\
Df & 28.00 & \\
t Stat & 1.46 & \\
P(T<=t) one-tail & $\mathbf{0 . 0 8}$ & \\
\hline
\end{tabular}

Table 4. Length of Stay

\begin{tabular}{|lrr|}
\hline Length of Stay (in days) & & \\
\hline & NON-Ofirmev & Ofirmev group \\
\hline Mean & 7.20 & $\mathbf{1 1 . 4 0}$ \\
Observations & 15.00 & 15.00 \\
Df & 28.00 & \\
t Stat & $\mathbf{2 . 4 3}$ & \\
$\mathbf{P}(\mathbf{T}<=\mathbf{t})$ one-tail & $\mathbf{0 . 0 1}$ & \\
\hline
\end{tabular}

Next, the summary and conclusions will be discussed. 


\section{Summary and Conclusions}

More than $80 \%$ of patients undergoing surgery experience post-operative pain, and $75 \%$ of those patients rate their pain as moderate, severe, or extreme (Chou et al., 2016). Cardiothoracic surgery is no different as it deploys a very invasive approach. Historically, cardiothoracic surgery has been treated post-operatively with various techniques and medications. For example, in a study by Rafiq et al. (2014), researchers administered ketorolac and dexamethasone once pre-operatively with scheduled ibuprofen, gabapentin, acetaminophen. The hope in this study was that if each medication targeted different pain pathways, there would be a reduction in pain reported, as well as morphine consumption. Kumar et al. (2018) also investigated alternative pain treatments by using a pectoral nerve block of a local anesthetic to decrease pain related to chest tubes. Pain management in surgical patients is unmistakably a research priority. There is a need for improvements in recovery and postsurgical adverse events such as nausea, vomiting, constipation, and ileus can be avoided (Kwan, 2017). The literature provides ample evidence that it is important to consider both combinations of medications, as well as alternative pain management techniques. The goal of decreasing pain after cardiac surgery and providing for the best patient outcomes is priority.

In discussing the many different techniques and medications available for pain management, it has been shown that integrating well-known medications with newer routes, such as inclusion of Ofirmev (IV acetaminophen), might be beneficial. As mentioned earlier, pain has many different pathways that can affect the body in numerous ways. The purpose of this study was to investigate if utilizing an existing pain 
management approach, along with the addition of Ofirmev, effected pain scores in postoperative cardiothoracic patients in a non-experimental retrospective chart review.

This study was a retrospective two group comparative chart review, which investigated pre and post implementation of an Ofirmev pain control protocol for cardiothoracic surgical patients. The sample for this project was derived from medical records, including both males and females, 18 years of age and older, in which cardiac surgery with full sternotomy was completed. The sample was separated into two groups of cardiac surgical patients. The interventional group, Group A, consisted 15 cardiothoracic surgical patients, post-implementation of the Ofirmev protocol for surgical pain management in September 2017. The standard group, Group B, was chosen from 15 cardiothoracic surgical patients from the year prior to Ofirmev availability. Data was collected pertaining to age, gender, opioid consumption for 24 hour period, pain scores at hour $0,6,10,16$, and 24 .

The results concluded that there were statistically significant differences in pain scores at hours 6 (Table 1) and at hour 24 (Table 2). At 6 hours, the non-ofirmev group reported significantly higher pain scores than that of the Ofirmev group (Table 1). Similarly, the non-Ofirmev group once again reported significantly higher pain scores than the Ofirmev group at the 24 hour mark. There was a lack of significance in the other time frames of the study. This is similar to the findings of Rafiq et al. (2014), who found post-operatively at 24 hours that the average pain score was significantly lower in a multimodal group $(\mathrm{M}=2.7 ; \mathrm{SD}=1.4)$ compared to a morphine only group $(\mathrm{M}=3.6$, $\mathrm{SD}=1.6)(p=0.005)$. 
The morphine equivalent consumption in the twenty-four hour period between the groups was not significant, but did approach statistical significance (Table 3). Although past cardiac surgical research has not shown evidence of total morphine equivalent consumption being measured, research into other surgical procedures has examined this. For example, Herring et al. (2014) evaluated multimodal treatment versus opioid only consumption in patients who had a total hysterectomy and found statistically significant results $(p=0.001)$ favoring less morphine use in the multimodal treatment group. These findings are comparable with the results in the current study and align with the benefits of using a multimodal medication regimen to manage pain in any surgical situation.

Results regarding the length of stay were contrary to the expectation that the length of stay in the Ofirmev group would be shorter than the non-ofirmev group. In studies involving other types of surgeries using Ofirmev, the groups receiving Ofirmev resulted in shorter, although not statistically significant, lengths of stay. For instance, a study done by Billings, Petracek, Roberts, \& Pretorius (2015) showed that the length of stay for patients on IV acetaminophen versus placebo was insignificant $(p<0.07)$ but approached significance for the IV acetaminophen group. Rafiq et al. (2014) found length of stay insignificantly lower in the multimodal group $(\mathrm{M}=7.4, \mathrm{SD}=3.3)$ versus morphine group ( $\mathrm{M}=8.2, \mathrm{SD}=4.6, p=0.257)$. In the current study, the length of stay in the nonofirmev group was found to be significantly lower than the Ofirmev group (Table 4). It is important to note that length of stay determination includes the entire stay, which may be skewed if a patient was admitted prior to the procedure.

The study was limited by the small sample size of 30 total charts and the choice of a retrospective design. During the chart review process, it was noted that there were many 
incomplete charts or patients who did not meet inclusion criteria, which restricted the size of this sample. It was found that numerous charts did not have complete pain score documentation for each of the intended hours. Additionally, as previously noted, length of stay may have included pre-procedure admissions, which may account for the greater length of stay of those in the non-ofirmev group. It may have been more helpful to retrieve only charts with admission on the day of surgery. One study by Peterson et al. (2002) showed that length of stay for those undergoing elective cardiac surgery ( $n=307,690 ; \mathrm{M}=6.6$ days) was shorter than those having emergent cardiac surgeries $(n=29,945 ; M=8.5$ days). Emergent cardiac surgeries often result due to secondary comorbid conditions, such as cardiogenic shock or failed attempt to stent a blocked vessel in a symptomatic patient, which may have a substantial impact on recovery and length of stay. If the current study had excluded emergency surgical patients, there may have similarly been a subsequent impact on the length of stay findings. Cardiothoracic surgery is a surgery that is unlike any other, especially in reference to pain experience, so these findings are not generalizable to those having non-cardiac procedures.

Pain after surgery that is mismanaged has been shown to promote complications in many post-operative patients. The theory of unpleasant symptoms marks how pain, an unpleasant symptom of surgery, can lead to post-operative complications, such as atelectasis, paralytic ileus, or pneumonia. The use of opioids alone have been found inadequate to manage post-operative pain and recovery, however in this current study, adding a medication, such as Ofirmev, which targets alternative pain receptors, was found to be effective in aiding post-operative pain while also decreasing morphine consumption. Although morphine consumption was not found to be significantly 
decreased in patients who received Ofirmev, there was less morphine consumed on average to this group. This research lends support to the use of Ofirmev in surgical patients, however it also demonstrates that there is a need for more study regarding the benefits of its use in other types of surgeries, as well as with a more diverse patient group. 


\section{Recommendations and Implications for Advanced Nursing Practice}

High levels of pain associated with open heart surgery leads to patients consuming large amounts of opioids. This can be problematic for many reasons. First, opioids only block one pathway for pain. Second, many patients are fearful of opioids due to the opioid epidemic that is currently a priority in healthcare. The fear of addiction to opioids is more relevant today than ever. According to the Center for Disease Control and Prevention ([CDC], 2018), opioids have killed more than 63,632 people in 2016, with $66 \%$ of those deaths involving prescription opioids or an illicit drug. The opioid crisis in the United States exerts pressure on healthcare to diminish the quantity of opioid prescriptions. This has led to the exploration of using multimodal pain management regimens to assist in better pain outcomes.

As prescribers, APRNs need to be aware of the different types of pain, their causes, and ways to minimize the pain. This study shows that using Ofirmev may decrease pain, including in a post-operative population. APRNs need to move from monotherapy to multi-modal therapy in targeting pain. It is also important to address opioid concerns that patients may express, including fear of addiction. This study shows that there is potential to decrease opioid consumption when adding Ofirmev to opioid treatment for pain in cardiac surgery. Not only does the addition of Ofirmev decrease opioid consumption, this study also demonstrated that pain scores may be decreased. Rafiq et al. (2014) similarly found that adding pain medications with multiple pain pathway targets provides better pain relief.

During the chart review it was noted that many records had to be omitted due to lack of pain score recordings by the clinical nurse. Therefore, the APRN can take this 
opportunity to teach the importance of documenting hourly pain scores and suggest alternate pain management techniques, such as Ofirmev. In reviewing the charts, there were many high pain scores that were as high as ten on the numbers rating scale, indicating mismanagement of pain. The APRN needs to teach the importance of minimizing pain to avoid pain at these levels. Through education the APRN can support clinical nurses by presenting evidence with current research on pain management in a multi-modal approach.

All surgical patients suffer from some degree of pain that needs to be addressed in the post-operative period. It is important for those in direct care of these patients to adequately manage pain. Utilizing different classes of medications to target specific pathways is important to patient outcomes in targeting different pain pathways, such as opioids targeting mu, kappa, and delta opioid receptors, whereas acetaminophen acts to block cyclooxygenase enzymes and prostaglandins (Douzjian \& Kulik, 2017). In one cardiothoracic study, Rafiq et al. (2014) showed that administering ketorolac, paracetamol (acetaminophen), gabapentin, ibuprofen, and dexamethasone had significant results of lower pain scores compared to just giving morphine and paracetamol only. The research by Rafiq et al. illustrates how the use of different adjuvants can decrease pain and increase positive outcomes. The APRN first needs to acknowledge that multi-modal medicinal approaches are a great improvement over opioid-only. Then the APRN may appropriately implement multi modal combinations to decrease: opioid side effects, pain levels, and length of stay in their practice, thus allowing for enhanced recovery.

The research for managing pain in the cardiac surgery population is still expanding to explore techniques and adjuvant medications. Since there remains a lack of 
standardization in treatment for pain related to cardiac surgery, perhaps future research will lead to a universal approach. This study highlights the need to utilize alternative medications in conjunction with opioids.

In summary, pain management is a very diverse, complicated, and seemingly endless area for research. This study shows the need to explore alternative sources to decrease pain in surgery. APRNs can make an impact in the change that is needed to address post-operative pain in all surgical realms. It is important to continue to utilize different ways to decrease pain without the use of opioids as the epidemic continues. 


\section{References}

American Pain Society.(2018). Pain Monograph. Retrieved from:

http://americanpainsociety.org/search-results?q=pain\%20pathways

Apfel, C., Jahr, J.R., Kelly, C.L., Ang, R.Y., \& Oderda, G.M.(2015). Effect of IV acetaminophen on total hip or knee replacement surgery: A case-matched evaluation of a national patient database. American Journal of Health-System Pharmacy, 72, 1961-1968.

Arslan, M., Celep, B., Ciçek, R., Kalender, H. Ü., \& Y1lmaz, H. (2013). Comparing the efficacy of preemptive intravenous paracetamol on the reducing effect of opioid usage in cholecystectomy. Journal of Research in Medical Sciences: The Official Journal of Isfahan University of Medical Sciences, 18(3), 172-177.

Babaee, S., Shafiei, Z., Sadeghi, M. M. M., Nik, A. Y., \& Valiani, M. (2012).

Effectiveness of massage therapy on the mood of patients after open-heart surgery. Iranian Journal of Nursing and Midwifery Research, 17(2 Supp11), S120.

Billings IV, F. T., Petracek, M. R., Roberts II, L. J., \& Pretorius, M. (2015). Perioperative intravenous acetaminophen attenuates lipid peroxidation in adults undergoing cardiopulmonary bypass: a randomized clinical trial. PloS one, 10(2), e0117625.

Center for Disease Control. ([CDC], 2018). U.S. drug overdose deaths continue to rise; increase fueled by synthetic opioids. Retrieved from https://www.cdc.gov/media/releases/2018/p0329-drug-overdose-deaths.html 
Chou, R., Gordon, D.B., Leon-Casasola, O.A., Rosenberg, J.M, Bickler, S., Brennan, T.,.. Wu, C.L..(2016). Management of postoperative pain: A Clinical Practice Guideline from the American Pain Society, the American Society of Regional Anesthesia and Pain Medicine, \& the American Society of Anesthesiologists' Committee on Regional Anesthesia, Executive Committee, and Administrative Council. The Journal of Pain: Official Journal of the American Pain Society. 17(2) $131-157$.

Douzjian, D. J., \& Kulik, A. (2017). Old drug, new route: a systematic review of intravenous acetaminophen after adult cardiac surgery. Journal of Cardiothoracic and Vascular Anesthesia, 31(2), 694-701.

Drugs.com(2018) Ketorolac. Retrieved from: https://www.drugs.com/pro/ketorolac.html

Drugs.com(2018) Morphine. Retrieved from:

https://www.drugs.com/search.php?searchterm=morphine+sulfate

Drugs.com(2019) Ofirmev. Retrieved from:

https://www.drugs.com/search.php?searchterm=ofirmev

Fot, E. V., Izotova, N. N., Yudina, A. S., Smetkin, A. A., Kuzkov, V. V., \& Kirov, M. Y. (2017). Automated weaning from mechanical ventilation after off-pump coronary artery bypass grafting. Frontiers in Medicine, 4, 31.

French, C.L., Crawford, S.L., Bova, C., \& Irwin, R. S. (2017). Change in psychological, physiological, and situational factors in adults after treatment of chronic cough. CHEST , $152,547-562$. 
Gebhart, G. F. (2000). Scientific issues of pain and distress. In Definition of Pain and Distress and Reporting Requirements for Laboratory Animals: Proceedings of the Workshop Held June 22, 2000. National Academies Press (US).

Herring, B. O., Ader, S., Maldonado, A., Hawkins, C., Kearson, M., \& Camejo, M. (2014). Impact of intravenous acetaminophen on reducing opioid use after hysterectomy. Pharmacotherapy, 34 Suppl 127S-33S. doi:10.1002/phar.1513

Izrailtyan, I., Qiu, J., Overdyk, F. J., Erslon, M., \& Gan, T. J. (2018). Risk factors for cardiopulmonary and respiratory arrest in medical and surgical hospital patients on opioid analgesics and sedatives. PloS one, 13(3), e0194553.

Kumar, K. N., Kalyane, R. N., Singh, N. G., Nagaraja, P. S., Krishna, M., Babu, B., ... Varadaraju. (2018). Efficacy of bilateral pectoralis nerve block for ultrafast tracking and postoperative pain management in cardiac surgery. Annals of Cardiac Anaesthesia, 21(3), 333.

Kwan, T. M. (2017). The Use of Intravenous Ibuprofen and Intravenous Acetaminophen in Surgical Patients and the Effect on Opioid Reduction. MEDSURG Nursing, $26(2), 124$.

Mayo Clinic.(2018). Intestinal Obstruction. Retrieved from: https://www.mayoclinic.org/diseases-conditions/intestinal-obstruction/symptomscauses/syc-20351460 
Mohamad, A., McDonnell, N., Bloor, M., Nathan, E., \& Paech, M. (2014). Parecoxib and paracetamol for pain relief following minor day-stay gynaecological surgery. Anaesthesia \& Intensive Care, 42(1), 43-50.

Murnion, B. P. (2018). Neuropathic pain: current definition and review of drug treatment. Australian Prescriber, 41(3), 60-63.

Nachiyunde, B., \& Lam, L. (2018). The efficacy of different modes of analgesia in postoperative pain management and early mobilization in postoperative cardiac surgical patients: A systematic review. Annals of Cardiac Anaesthesia, 21(4), 363-370.

Peterson, E. D., Coombs, L. P., Ferguson, T. B., Shroyer, A. L., DeLong, E. R., Grover, F. L., ... \& STS National Cardiac Database Investigators. (2002). Hospital variability in length of stay after coronary artery bypass surgery: results from the Society of Thoracic Surgeon's National Cardiac Database. The Annals of Thoracic Surgery, 74(2), 464-473.

Polit, D., \& Beck, C. (2017). Nursing research generating and assessing evidence for nursing practice. New York: Wolters Kluwer.

Rafiq, S., Steinbrüchel, D. A., Wanscher, M. J., Andersen, L. W., Navne, A., Lilleoer, N. B., \& Olsen, P. S. (2014). Multimodal analgesia versus traditional opiate based analgesia after cardiac surgery, a randomized controlled trial. Journal of Cardiothoracic Surgery, 9(1), 52-59. 
Salani, D., Crenshaw, N. A., Owusu, B., \& Gonzalez, J. M. (2018). Pain management in an opioid epidemic: What's Appropriate, What's Safe. Clinician Reviews, 28(4), 40-47. Retrieved from: http://search.ebscohost.com.ric.idm.oclc.org/login.aspx?direct=true $\& d b=a 9 h \& A N$ $=129477566 \&$ site $=$ ehost-live

Smith, M. J., \& Liehr, P. R. (Eds.). (2018). Middle range theory for nursing. Springer Publishing Company.

Strode, M. A., Sherman, W., Mangieri, C. W., Bland, C. M., Sparks, P. J., Faler, B. J., \& ... Choi, Y. U. (2016). Randomized trial of OFIRMEV versus placebo for pain management after laparoscopic sleeve gastrectomy. Surgery for Obesity and Related Diseases: Official Journal of The American Society for Bariatric Surgery, 12(4), 772-777.

Waknine, Y. (2010). FDA Approves first intravenous formulation of acetaminophen. Medscape. Retrieved from: https://www.medscape.com/viewarticle/731994

Woods, S. J., Kozachik, S. L., \& Hall, R. J. (2010). Subjective sleep quality in women experiencing intimate partner violence: Contributions of situational, psychological, and physiological factors. Journal Of Traumatic Stress, 23(1), 141-150.

Zubrzycki, M., Liebold, A., Skrabal, C., Reinelt, H., Ziegler, M., Perdas, E., \& Zubrzycka, M. (2018). Assessment and pathophysiology of pain in cardiac surgery. Journal of Pain Research, 11, 1599. 
Appendix A

\begin{tabular}{|c|c|c|c|c|c|c|c|c|c|}
\hline No. & Age & IVA & MEQ & P0 & P6 & P10 & P16 & P24 & LOS \\
\hline & & & & & & & & & \\
\hline & & & & & & & & & \\
\hline & & & & & & & & & \\
\hline
\end{tabular}

No. $=$ Subject ID ;IVA = Intravenous Acetaminophen; MEQ = Morphine Equivalent; $\mathrm{P} 0=$ Pain score at 0 Hours, $\mathrm{P} 6=$ Pain score at 6 hours, $\mathrm{P} 10=$ Pain score at 10 hours, P16=Pain score 16 hours, P24= Pain score at 24 hours, LOS=Length of Stay 